\title{
Field-dependent ionic conductivities from generalized fluctuation-dissipation relations
}

\author{
Dominika Lesnicki, ${ }^{1}$ Chloe Y. Gao, ${ }^{2}$ Benjamin Rotenberg,,${ }^{1,3}$ and David T. Limmer ${ }^{2,4,5,6, \text { * }}$ \\ ${ }^{1}$ Sorbonne Université, CNRS, Physico-Chimie des électrolytes et Nanosystèmes Interfaciaux, F-75005 Paris, France \\ ${ }^{2}$ Department of Chemistry, University of California, Berkeley, California \\ ${ }^{3}$ Réseau sur le Stockage Electrochimique de l'Energie (RS2E), FR CNRS 3459, France \\ ${ }^{4}$ Kavli Energy NanoScience Institute, Berkeley, California \\ ${ }^{5}$ Materials Science Division, Lawrence Berkeley National Laboratory, Berkeley, California \\ ${ }^{6}$ Chemical Science Division, Lawrence Berkeley National Laboratory, Berkeley, California
}

(Dated: March 20, 2020)

\begin{abstract}
We derive a relationship for the electric field dependent ionic conductivity in terms of fluctuations of time integrated microscopic variables. We demonstrate this formalism with molecular dynamics simulations of solutions of differing ionic strength with implicit solvent conditions and molten salts. These calculations are aided by a novel nonequilibrium statistical reweighting scheme that allows for the conductivity to be computed as a continuous function of the applied field. In strong electrolytes, we find the fluctuations of the ionic current are Gaussian and subsequently the conductivity is constant with applied field. In weaker electrolytes and molten salts, we find the fluctuations of the ionic current are strongly non-Gaussian and the conductivity increases with applied field. This nonlinear behavior, known phenomenologically for dilute electrolytes as the Onsager-Wien effect, is general and results from the suppression of ionic correlations at large applied fields, as we elucidate through both dynamic and static correlations within nonequilibrium steady-states.
\end{abstract}

Advances in the fabrication of nanofluidic devices have enabled the study of transport processes on small scales, where novel phenomena emerge from the interplay of confinement, fluctuations and molecular granularity 14 Some of the most striking recent observations have been in electrokinetic transport of electrolyte solutions confined to nanometer dimensions. In such systems, large thermodynamic gradients can be generated, driving nonlinear responses such as Coulomb blockade and current rectification.5 ${ }^{[5]}$ At the same time, and independently, theoretical developments have permitted the application of response theory to systems far from equilibrium. In particular, generalized fluctuation-dissipation theorems have been formulated for linear responses of nonequilibrium steady states, or nonlinear responses around equilibrium states $10-16$ These coincident developments provide a way for predicting transport relationships from molecular properties, and using such relationships to design nanofluidic devices. Building on these previous work, and inspired by emerging challenges in nanofluidic devices, we develop a theory and accompanying numerical technique to efficiently compute the electric field dependent conductivity in ionic solutions. We recover behavior similar to the Onsager-Wien effect in the dilute limit, 17 however our calculations are valid across all concentration regimes, and arbitrary nonlinear responses. Our approach is general and can be extended to other systems or transport processes where a connection between nonlinear transport behavior and underlying microscopic dynamics is desired.

We consider a system of $N$ ions composed of $N_{a}$ anions and $N_{c}$ cations, in a volume $V$ and fixed temperature, $T$. The ions' positions and velocities are denoted, $\mathbf{r}^{N}=\left\{\mathbf{r}_{1}, \mathbf{r}_{2}, \ldots, \mathbf{r}_{N}\right\}$ and $\mathbf{v}^{N}=\left\{\mathbf{v}_{1}, \mathbf{v}_{2}, \ldots, \mathbf{v}_{N}\right\}$, respectively. These variables evolve according to an under- damped Langevin equation,

$$
\dot{\mathbf{x}}_{i}=\mathbf{v}_{i}, \quad m_{i} \dot{\mathbf{v}}_{i}=-\zeta_{i} \mathbf{v}_{i}+\mathbf{F}_{i}\left(\mathbf{r}^{N}\right)+z_{i} \mathbf{E}+\boldsymbol{\eta}_{i}
$$

where $m_{i}$ and $z_{i}$ are the $i$ th particle's mass and charge, $\zeta_{i}$ is the friction from the implicit solvent, and $\mathbf{F}_{i}\left(\mathbf{r}^{N}\right)$ is the interparticle force on ion $i$, which we take as a pairwise sum of electrostatic interaction with dielectric constant $\epsilon_{s}$ and non-electrostatic (short-range repulsion and long-range dispersion) forces. Further details of the force fields can be found in the Supplemental Material (SM). Each cartesian component of the random force, $\eta_{i \alpha}$, obeys Gaussian statistics with mean $\left\langle\eta_{i \alpha}\right\rangle=0$ and variance $\left\langle\eta_{i \alpha}(t) \eta_{j \beta}\left(t^{\prime}\right)\right\rangle=2 k_{\mathrm{B}} T \zeta_{i} \delta_{i j} \delta_{\alpha \beta} \delta\left(t-t^{\prime}\right)$, where $k_{\mathrm{B}}$ is Boltzmann's constant. Finally, $\mathbf{E}$ denotes an applied electric field, with magnitude $E$, which drives an ionic current through the periodically replicated system. This equation of motion does not conserve momentum, and thus hydrodynamic effects are explicitly neglected throughout.

To compute the ionic conductivity as a function of electric field, we aim to relate dynamic quantities of the system at a reference field, to those of a system perturbed by an additional applied field. Given the equation of motion in Eq. 1. the probability of observing a trajectory, $\mathbf{X}\left(t_{\mathrm{N}}\right)$, or sequence of positions and velocities over an observation time, $t_{\mathrm{N}}$, with an applied field, is

$$
P_{\mathbf{E}}\left[\mathbf{X}\left(t_{\mathrm{N}}\right)\right] \propto e^{-\beta U_{\mathbf{E}}\left[\mathbf{X}\left(t_{\mathrm{N}}\right)\right]}
$$

where for the uncorrelated Gaussian noise, we have an Onsager-Machlup stochastic action $\frac{18}{18}$ of the form

$$
U_{\mathbf{E}}\left[\mathbf{X}\left(t_{\mathrm{N}}\right)\right]=\sum_{i=1}^{N} \int_{0}^{t_{\mathrm{N}}} d t \frac{\left[m_{i} \dot{\mathbf{v}}_{i}+\zeta_{i} \mathbf{v}_{i}-\mathbf{F}_{i}\left(\mathbf{r}^{N}\right)-z_{i} \mathbf{E}\right]^{2}}{4 \zeta_{i}}
$$


where the stochastic calculus is interpreted in the Itô sense. We will consider trajectories in the limit that $t_{\mathrm{N}}$ is large so that only time extensive quantities are relevant.

The addition of a perturbing field on the system adds an extra drift to the Gaussian action. As a consequence, we can write the ratio of the probability to observe a trajectory in the presence of the field, $\mathbf{E}=\mathbf{E}_{r}+\Delta \mathbf{E}$, relative to the probability to observe a trajectory with $\mathbf{E}_{r}$,

$$
\frac{P_{\mathbf{E}_{r}+\Delta \mathbf{E}}\left[\mathbf{X}\left(t_{\mathrm{N}}\right)\right]}{P_{\mathbf{E}_{r}}\left[\mathbf{X}\left(t_{\mathrm{N}}\right)\right]}=e^{\beta \Delta U_{\Delta \mathbf{E}}\left[\mathbf{X}\left(t_{\mathrm{N}}\right)\right]}
$$

where the dimensionless relative action, $\beta \Delta U_{\Delta \mathbf{E}}\left[\mathbf{X}\left(t_{\mathrm{N}}\right)\right]$, can be expressed compactly as a sum of three terms, depending on their symmetry under time reversal,, 16

$$
\frac{\Delta U_{\Delta \mathbf{E}}}{t_{\mathrm{N}}}=\left[J+Q-E_{r} \sigma_{\mathrm{id}} V\right] \frac{\Delta E}{2}-\sigma_{\mathrm{id}} V \frac{\Delta E^{2}}{4}
$$

where for simplicity we take the field along one cartesian direction so that the relative action depends only on its magnitude. The first term is asymmetric under time reversal and identified as the excess entropy production due to the increased nonequilibrium driving. It is given by the product of the total, time averaged ionic current in the direction of the field,

$$
J\left[\mathbf{X}\left(t_{\mathrm{N}}\right)\right]=\frac{1}{t_{\mathrm{N}}} \int_{0}^{t_{\mathrm{N}}} d t j(t), \quad j(t)=\sum_{i=1}^{N} z_{i} v_{i}(t)
$$

and the extra field $\Delta E / 2$. The second term in Eq. 5 is symmetric under time reversal and referred to as the excess frenesy, ${ }^{[19}$ where

$$
\begin{aligned}
Q\left[\mathbf{X}\left(t_{\mathrm{N}}\right)\right] & =\frac{1}{t_{\mathrm{N}}} \int_{0}^{t_{\mathrm{N}}} d t q(t), \\
q(t) & =\sum_{i=1}^{N} \frac{z_{i}}{\zeta_{i}}\left[m_{i} \dot{v}_{i}(t)-F_{i}\left(\mathbf{r}^{N}\right)\right]
\end{aligned}
$$

includes the total time integrated force in the direction of the field weighted by $z_{i} / \zeta_{i}$, and a boundary term resulting in a difference in velocities at times 0 and $t_{\mathrm{N}}$, times the extra field. The remaining terms are trajectory independent constants, which are proportional to

$$
\sigma_{\mathrm{id}}=\frac{N_{c} z_{c}^{2} D_{c}+N_{a} z_{a}^{2} D_{a}}{V k_{\mathrm{B}} T}
$$

which is the Nernst-Einstein conductivity of the solution, where $D_{i}=k_{\mathrm{B}} T / \zeta_{i}$ is the diffusion coefficient for an isolated ion of type $i$. This decomposition of the relative action admits particularly simple, physically transparent, nonlinear response relations 12

With the relative measure between trajectory ensembles defined in Eq. 4, we can follow previous work by Gao and Limmer, $\frac{16}{16}$ and relate nonequilibrium trajectory averages in the presence of the field, to equilibrium trajectory averages in the absence of the field. We do this by setting the reference field, $E_{r}=0$, so that $E=\Delta E$. For a trajectory observable, $O\left[\mathbf{X}\left(t_{\mathrm{N}}\right)\right]$, this relation is

$$
\langle O\rangle_{E}=\left\langle O e^{\beta \Delta U_{\mathbf{E}}\left[\mathbf{X}\left(t_{\mathrm{N}}\right)\right]}\right\rangle_{0}
$$

where trajectory averages over the measure in Eq. 2, with field value $E$, are denoted $\langle\ldots\rangle_{E}$. Setting $O$ to 1 , we find a sum rule inherited from the underlying Gaussian process that is quadratic in the field,

$$
\left\langle e^{\beta t_{\mathrm{N}}\left(J\left[\mathbf{X}\left(t_{\mathrm{N}}\right)\right]+Q\left[\mathbf{X}\left(t_{\mathrm{N}}\right)\right]\right) E / 2}\right\rangle_{0}=e^{\beta t_{\mathrm{N}} \sigma_{\mathrm{id}} V E^{2} / 4}
$$

which is interpretable as the ratio of nonequilibrium to equilibrium trajectory partition functions.

Identifying the joint probability of observing a value of the current and frenesy as $p_{E}(J, Q)=\langle\delta(J-$ $\left.\left.J\left[\mathbf{X}\left(t_{\mathrm{N}}\right)\right], Q-Q\left[\mathbf{X}\left(t_{\mathrm{N}}\right)\right]\right)\right\rangle_{E}$, we can relate $p_{E}(J, Q)$ to its equilibrium counterpart, using Eq. 9 ,

$$
\frac{\ln p_{0}(J, Q)}{t_{\mathrm{N}}}=\frac{\ln p_{E}(J, Q)}{t_{\mathrm{N}}}-\beta(J+Q) \frac{E}{2}+\beta \sigma_{\mathrm{id}} V \frac{E^{2}}{4}
$$

where we find that the nonequilbrium driving acts to reweight the joint distribution linearly in $J+Q$, demonstrating a thermodynamic-like relationship between this sum and its conjugate quantity $E$. Note that such linearity is not in general valid for the marginal distribution of just the current, $p_{0}(J)=\int d Q p_{0}(J, Q)$, due to correlations between $J$ and $Q$. Equation 11 provides a route to numerically probe the tails of nonequilibrium probability distributions using generalizations of histogram reweighing techniques, such as those developed for equilibrium systems, like multicanonical sampling. 20

With access to the joint distribution, $p_{0}(J, Q)$, we can compute the relationship between the mean current and the applied electric field arbitrarily far from equilibrium, as encoded in the electric field dependent conductivity, $\sigma(E)=\left(d\langle J\rangle_{E} / d E\right) / V$. Using Eq. 9 to first write the average current density, and then differentiating with respect to the field, we find

$$
\sigma(E)=\lim _{t_{\mathrm{N}} \rightarrow \infty} \frac{\beta t_{\mathrm{N}}}{2 V}\left\langle\left(\delta J^{2}+\delta J \delta Q\right) e^{\beta \Delta U_{\mathbf{E}}[\mathbf{X}]}\right\rangle_{0}
$$

where $\delta O=O-\langle O\rangle$, demonstrating that $\sigma(E)$ is given by a sum of the variance of the current and the currentfrenesy correlations, reweighted by the factor that relates the equilibrium average to the nonequilibrium ensemble at fixed $E$. Near equilibrium $(E \approx 0)$, the weight $\approx$ 1 , and fluctuations in $J$ and $Q$ are uncorrelated due to the time reversal invariance of detailed balance dynamics, $\langle\delta J \delta Q\rangle_{0}=0$. In this limit Eq. 12 reduces to a standard Einstein-Helfand relationship $\frac{21}{21}$ For small values of $E$, we can expand the weight, and the first non-vanishing term emerges at second order in the field $\frac{16}{16}$ and vanishes for uncorrelated Gaussian random variables. ${ }^{22}$ 

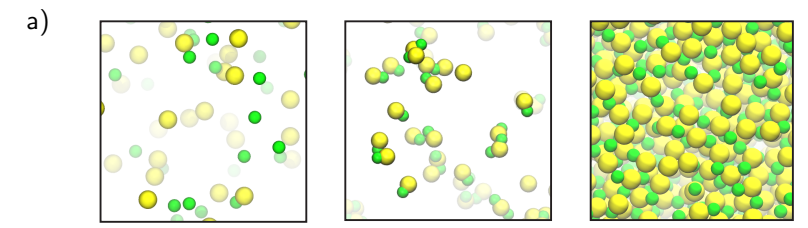

b)
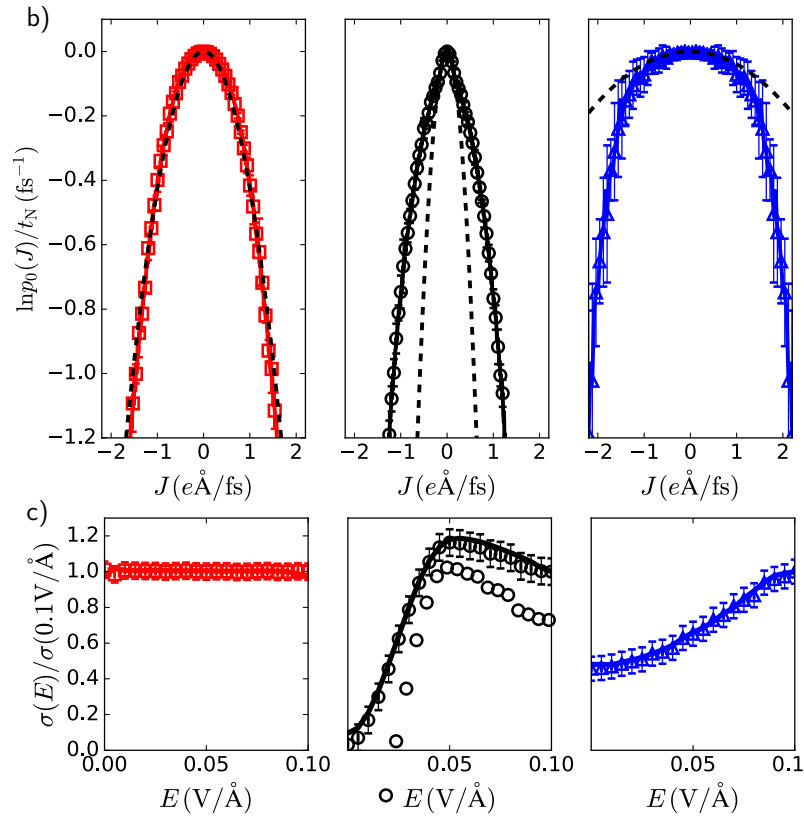

$J(e \AA / \mathrm{fs})$

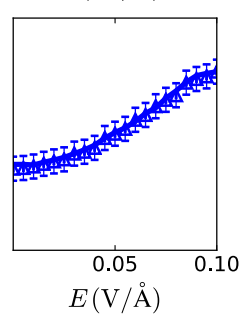

FIG. 1. Fluctuations and response for the $0.1 \mathrm{M}$ solution of $\mathrm{NaCl}$ with $\epsilon_{s}=78.5$ (left) and $\epsilon_{s}=10$ (middle), and the 25.3 M molten $\mathrm{NaCl}$ with particle number $N$. a) Characteristic snapshots of the $\mathrm{NaCl}$ systems considered, with green and yellow spheres representing $\mathrm{Na}^{+}$and $\mathrm{Cl}^{-}$respectively. b) Scaled log-probability of the time integrated current as computed from histogram reweighting. Errorbars are one standard deviation of the mean as computed from bootstrapping analysis. The dashed lines represent Gaussian distributions with the same mean and variance. c) Field dependent ionic conductivities relative to their values at $E=0.1 \mathrm{~V} / \AA$. Lines are computed from reweighting $p(J, Q)$, and symbols are computed from finite differences of $\langle J\rangle_{E}$ versus $E$. Errorbars are one standard deviation of the mean.

We have used these formal relationships to study the electric field dependent conductivity of a number of different model systems. Specifically, we have studied simple electrolytes in implicit solvent with dielectric constants $\epsilon_{s}=10$ and 78.5 at $T=300 \mathrm{~K}$, and a molten salt with $\epsilon_{s}=1$ at $T=1200 \mathrm{~K}$. The main text presents results for $\mathrm{NaCl}$ at concentrations of $0.1 \mathrm{M}$, and $25.3 \mathrm{M}$ corresponding to a molten salt. These are illustrated in Fig. 17), while the SM presents additional results for $\mathrm{NaCl}$ and $\mathrm{MgCl}_{2}$ at $1.0 \mathrm{M}$. Frictions are taken to be $\zeta_{i}=m_{i} / \tau_{i}$ with $\tau_{c}=0.11 \mathrm{ps}$ for the cations and $\tau_{a}=0.25$ ps for the anions, and we find $t_{\mathrm{N}}=0.2 \mathrm{ps}$ is sufficient to converge the conductivity for the electrolyte system, while $t_{\mathrm{N}}=0.05 \mathrm{ps}$ is sufficient for the molten salt with $\tau_{c}=\tau_{a}=1.2 \mathrm{ps} \cdot{ }^{23}$ For the electrolyte solution the system size corresponds to $N_{c}=100$, and for the molten salt $N_{c}=2500$. Long-ranged electrostatic interactions are computed using Ewald summation, and all simulations are performed with the LAMMPS package. 24

Shown in Fig. 1b) are the current distributions computed from nonequilibrium molecular dynamics simulations for $E$ between 0 and $0.1 \mathrm{~V} / \AA$ in steps of $0.01 \mathrm{~V} / \AA$ combined using Eq. 11, followed by marginalization over $Q$. We find that for the dilute solution of $\mathrm{NaCl}$ with $\epsilon_{s}=78.5$, the current distribution is Gaussian. The Gaussian statistics follow from the largely dissociated nature of the strong electrolyte in the polar, implicit solvent, which enables ions to move free of correlations from their surrounding environment. Analogous Gaussian fluctuations are found for $1 \mathrm{M} \mathrm{NaCl}$ and $\mathrm{MgCl}_{2}$ with $\epsilon_{s}=78.5 .23$ This is in contrast to calculations with $\epsilon_{s}=10$, where ionic correlations depress motions, leading to smaller characteristic current fluctuations near equilibrium, as computed by its variance, $t_{\mathrm{N}}\left\langle J^{2}\right\rangle_{0}$. Weaker electrolyte systems exhibit marked deviations from Gaussian statistics with enhanced probability at large values of $J$. Similar behavior is found for $1 \mathrm{M} \mathrm{NaCl}$ and $\mathrm{MgCl}_{2}$ with $\epsilon_{s}=10 \cdot{ }^{23}$ The molten salt also exhibits deviations from Gaussian statistics but with narrow tails, signifying that fluctuations are much rarer than would be expected from its large variance. The narrow distribution reflects the packing constraints that inhibit large currents.

Shown in Fig. 1k) are the conductivities computed from $p_{0}(J, Q)$ continuously as a function of the applied field. We have additionally computed the conductivity from a numerical derivative of the average current versus applied field and find quantitative agreement between both estimates, although the statistical errors are much larger from the finite difference approach at fixed computational cost. For strong electrolytes that exhibit Gaussian current fluctuations, we find a field-independent conductivity, while both the weak electrolytes and molten salt that exhibit non-Gaussian current fluctuations have conductivities that increase with applied field. The increase is initially quadratic, as observed experimentally $\sqrt{25}$ for dilute solutions and necessitated by time-reversal symmetry, and plateaus at large fields. For the dilute solution the conductivity plateaus to the same value as the strong electrolytes. The plateau value is identified as the limit of uncorrelated motion, given by $\sigma_{\text {id }}$ in Eq. 8 . At intermediate fields, the dilute solution exhibits a slight maxima in conductivity as has been noted in colloids ${ }^{26}$ and low dimensional systems. ${ }^{[2]}$ The molten salt conductivity also increases and plateaus, though its plateau value is far below $\sigma_{\text {id }}$. This field dependence of the conductivity in Fig. 1 b) is phenomenologically known as the OnsagerWien effect in the dilute limit!1728

In order to understand the effect, we can unpack the relevant correlations using a generalized fluctuation dissipation relationship. Specifically, we rewrite the field- 

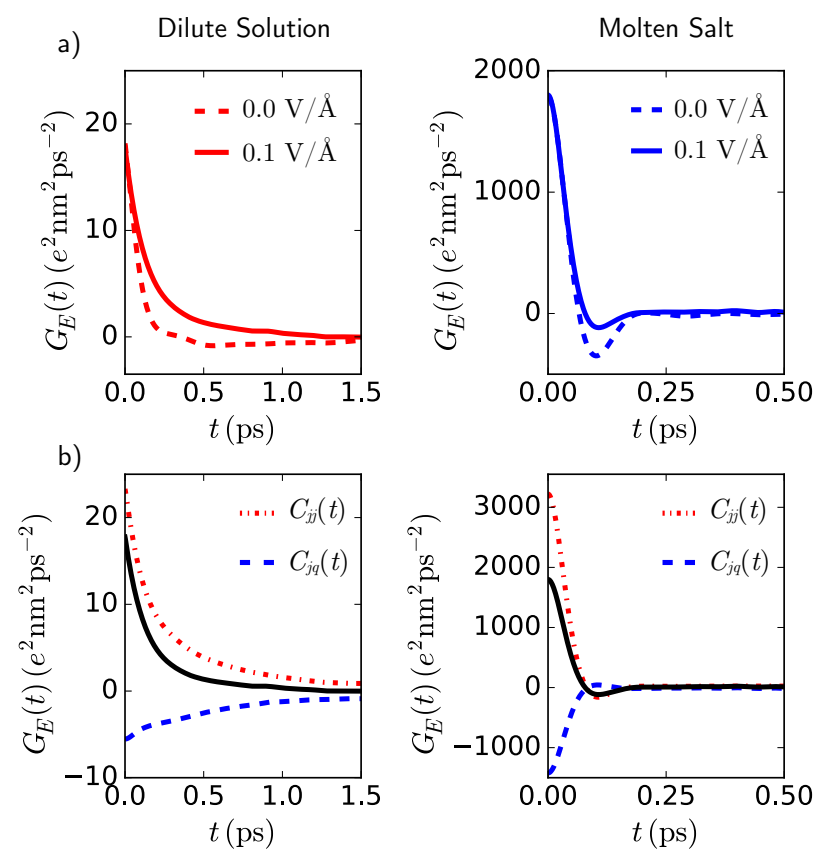

FIG. 2. Time correlation function $G_{E}(t)=C_{j j}(t)+C_{j q}(t)$ for the field dependent conductivity of $0.1 \mathrm{M} \mathrm{NaCl}$ with $\epsilon_{s}=10$ (left) and molten salt (right). a) Correlations functions for $E=0$ and $E=0.1 \mathrm{~V} / \AA$. b) Decomposition of $G_{E}(t)$ into current-current $C_{j j}(t)$ and current-frenesy $C_{j q}(t)$ correlations at $E=0.1 \mathrm{~V} / \AA$. The solid lines in b) are the total correlation functions.

dependent conductivity as an average within a nonequilibrium steady-state, using the same procedure by which we arrived at Eq. 12, only now within a trajectory ensemble at fixed $E$. In this case, the differential response of the current an applied field is

$$
\begin{aligned}
\sigma(E) & =\lim _{t_{\mathrm{N}} \rightarrow \infty} \frac{\beta t_{\mathrm{N}}}{2 V}\left\langle\left(\delta J^{2}+\delta J \delta Q\right)\right\rangle_{E} \\
& =\frac{\beta}{V} \int_{0}^{\infty} d t G_{E}(t)
\end{aligned}
$$

where $G_{E}(t)=C_{j j}(t)+C_{j q}(t), C_{j j}(t)=\langle\delta j(0) \delta j(t)\rangle_{E}$, $C_{j q}(t)=\frac{1}{2}\langle\delta j(0) \delta q(t)+\delta j(0) \delta q(-t)\rangle_{E}$. The conductivity away from equilibrium is a sum of the integrated microscopic current-current correlation function and the integrated microscopic current-frenesy correlation function. To arrive at this expression, we have assumed that the correlation functions decay faster than $1 / t$, and invoked the time reversal properties of $j$ and $q$ together with the stationarity of the nonequilibrium steady-state, to eliminate one of the time integrals.

Fig. 2a) shows the total time correlation function for the conductivity, for both the dilute electrolyte and the molten salt with and without an applied field. In the absence of an applied field, the only nonvanishing contribution to $G_{E}(t)$ is the current-current correlation func- tion. For both dilute electrolyte and the molten salt, current correlations decay within $1 \mathrm{ps}$. The molten salt exhibits noticeable recoil effects evident in transient negative correlations at intermediate times. The dilute electrolyte exhibits transient negative correlations at intermediate times as well, though these are spread over a broader range of timescales. In both cases, the negative correlations are ionic relaxation effects that result from ion displacements that transiently distort the local electrostatic environment and generate a restoring force on the displaced ion from the compensating ionic cloud left behind ${ }^{29} 31$ At high fields, this negative correlation is suppressed, resulting in a larger integrated value of the correlation function, hence larger conductivity. While the time-correlation functions in principle depend on the frictions in the Langevin thermostat, for the small values employed in this study, the current is independent of the friction and the frenesy depends on the friction only though the explicit factors of $\zeta_{i}$ in Eq. $77^{23}$

In Fig. 2p) we show the decomposition of $G_{E}(t)$ into the current-current correlation function and the currentfrenesy correlation function for $E=0.1 \mathrm{~V} / \AA$. For both systems, the current-current correlation function decays slower at high fields than at $E=0$ and accounts for the largest contribution to the $G_{E}(t)$ integrand. For the dilute electrolyte, positive contributions to the currentcurrent correlation function between unlike charges give rise to the shallow maximum at intermediate fields. These correlations are expected to be quenched out by momentum transfer to an explicit solvent. The currentfrenesy correlations are negative and the dominant contribution to the frenesy is the total charge weighted force, which directly manifests the depression of the conductivity due to ionic correlations. The magnitude of the correlations in the molten salt are larger than the dilute electrolyte, reflecting its size extensive definition. The decay in the correlation function for the molten salt is nearly ten times faster, manifesting the dense system where the mean free path of a given ion is small. For higher dielectric constant systems, the current-frenesy correlations are negligible, signifying the lack of ion correlations, and as a result, time correlation functions are independent of fields.

In the dilute solution limit, Onsager provided a theory for the field-dependent conductivity that relies on approximating the distortion of the pair correlation functions in the presence of an applied field $!^{[17}$ In order to understand the structural origins of these dynamical effects and make contact with the previous work by Onsager, we can relate the field dependent conductivity to the change in the static ion correlations. By noting that within the steady state, the ions are force free on average, $\left\langle\dot{v}_{i}\right\rangle_{E}=0$, we can rearrange the equation of motion in Eq. 1 and insert it into Eq. 6. This yields the average current density 

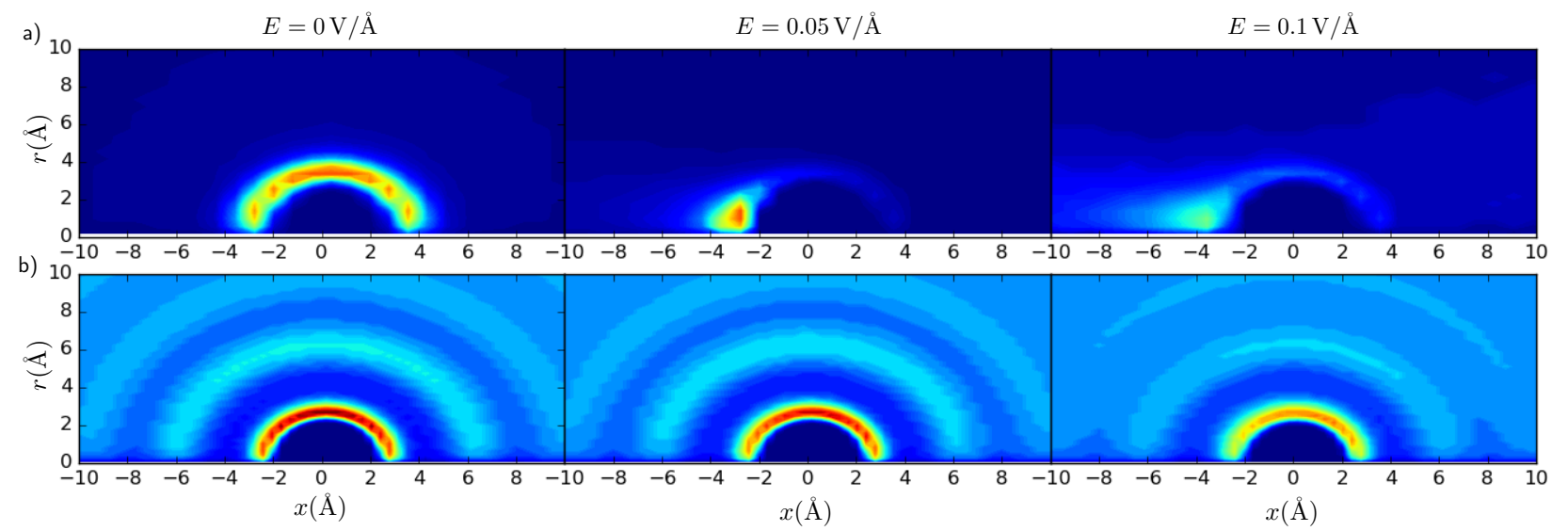

FIG. 3. Pair distribution functions, $g_{a, c}(\mathbf{r} \mid E)$, between $\mathrm{Cl}^{-}$and $\mathrm{Na}^{+}$with increasing field, plotted in the cylindrical coordinates for the a) dilute solution $0.1 \mathrm{M}, \epsilon_{s}=10$ and b) molten salt.

in the direction of the field,

$$
\frac{\langle J\rangle_{E}}{V}=\sigma_{\mathrm{id}} E+\sum_{i=c, a} \frac{N_{i}}{V} \frac{z_{i}}{\zeta_{i}}\left\langle F_{i}\left(\mathbf{r}^{N}\right)\right\rangle_{E}
$$

which is given by a sum of the Nernst-Einstein conductivity times the applied field, and a correlated contribution from the sum of the average force acting on ions weighted by their charge. We can express the average force in the direction of the finite field, with unit vector $\hat{\mathbf{x}}$, as

$$
\left\langle F_{i}\left(\mathbf{r}^{N}\right)\right\rangle_{E}=\sum_{j=c, a} \int d \mathbf{r} \rho_{j} g_{i, j}(\mathbf{r} \mid E) \hat{\mathbf{x}} \cdot \mathbf{F}_{i, j}^{(2)}(\mathbf{r})
$$

where $\rho_{j}$ is the number density of the $j$ th ion type, and we have introduced the pair distribution functions $g_{i, j}(\mathbf{r} \mid E)$ and the pairwise decomposable force, $\mathbf{F}_{i, j}^{(2)}$, between ions of type $i$ and $j$. The pair distribution function is defined as an average within the nonequilibrium steady-state

$$
g_{i, j}(\mathbf{r} \mid E)=\frac{1}{\rho_{i} \rho_{j}}\left\langle\sum_{k \in N_{i}, l \in N_{j}} \delta\left(\mathbf{r}_{k}\right) \delta\left(\mathbf{r}-\mathbf{r}_{l}\right)\right\rangle_{E}
$$

normalized by the product of the densities of $i$ and $j$. In the original Onsager treatment, Eq. 14 is assumed to have the form, $\langle J\rangle_{E} / V=\left[\sigma_{\mathrm{id}}+\Delta \sigma(E)\right] E$, where $\Delta \sigma(E)$ is the correlated contribution to the conductivity computable from the knowledge of how the pair distribution function changes with applied field.

Shown in Fig. 3 3 ) are the pair distribution functions between $\mathrm{Na}^{+}$and $\mathrm{Cl}^{-}$for $0.1 \mathrm{M}$ and $\epsilon_{s}=10$, and in Fig. 3p) for the molten salt, as a function of increasing applied field. In the presence of the field, the correlations deviate from spherical symmetry. As a consequence, we plot $g_{i, j}(\mathbf{r} \mid E)$ as a function of distance in direction of the applied field, $x$, and orthogonal radial coordinate, $r$, as the correlations do retain cylindrical symmetry. With increasing field, the correlations are found to distort away from spherical symmetry, polarizing in the direction of the applied field. This is more evident in the dilute solution compared to the molten salt. At large applied fields, the amplitude of the correlations decrease dramatically for the dilute solution, clarifying the limit of uncorrelated motion noted in Fig. 1p). Within the molten salt, correlations persist, as even large fields are insufficient to mitigate packing constraints.

In conclusion, we have leveraged recent developments in the theory of nonequilibrium systems to relate ionic conductivities to microscopic correlations under arbitrarily large electric fields. We have found that both the fluctuations of the ion's displacement as well as the dynamical fluctuations of the intrinsic electric fields acting on an ion, affect the response of the ionic current to an additional external field. This additional contribution is absent in the Green-Kubo expression for the conductivity near-equilibrium.21 This approach of reweighting nonequilibrium trajectories is general, and we expect will find use more broadly in other cases of molecular transport. It will be particularly interesting to apply these new statistical tools to investigate the nonlinear response of ionic liquids,,$\sqrt[32133]{ }$ as well as transport near charged interfaces, such as nonlinear electrofriction on corrugated surfaces ${ }^{34}$ or nonlinear electro-osmotic response.

Acknowledgments The authors thank Lydéric Bocquet for useful discussions. DL and BR acknowledge financial support from the French Agence Nationale de la Recherche (ANR) under Grant No. ANR-17-CE09-004602 (NEPTUNE). CYG was supported by the U.S. Department of Energy, Office of Basic Energy Sciences through Award Number de-sc0019375. BR and DTL were supported by the France-Berkeley Fund from the University of California, Berkeley. 
* dlimmer@berkeley.edu

[1] A. Esfandiar, B. Radha, F. Wang, Q. Yang, S. Hu, S. Garaj, R. Nair, A. Geim, and K. Gopinadhan, Science 358, 511 (2017).

[2] T. Mouterde, A. Keerthi, A. Poggioli, S. Dar, A. Siria, A. Geim, L. Bocquet, and B. Radha, Nature 567, 87 (2019).

[3] A. Siria, P. Poncharal, A.-L. Biance, R. Fulcrand, X. Blase, S. T. Purcell, and L. Bocquet, Nature 494, 455 (2013).

[4] E. Secchi, A. Niguès, L. Jubin, A. Siria, and L. Bocquet, Physical review letters 116, 154501 (2016).

[5] S. J. Kim, Y.-C. Wang, J. H. Lee, H. Jang, and J. Han, Physical review letters 99, 044501 (2007).

[6] R. Karnik, C. Duan, K. Castelino, H. Daiguji, and A. Majumdar, Nano letters 7, 547 (2007).

[7] W. Guan, R. Fan, and M. A. Reed, Nature communications 2, 506 (2011).

[8] U. Vermesh, J. W. Choi, O. Vermesh, R. Fan, J. Nagarah, and J. R. Heath, Nano letters 9, 1315 (2009).

[9] J. Feng, K. Liu, M. Graf, D. Dumcenco, A. Kis, M. Di Ventra, and A. Radenovic, Nature materials 15, 850 (2016).

[10] T. Speck and U. Seifert, EPL (Europhysics Letters) 74, 391 (2006).

[11] J. Prost, J.-F. Joanny, and J.M.R. Parrondo, Physical review letters 103, 090601 (2009).

[12] M. Baiesi, C. Maes, and B. Wynants, Physical review letters 103, 010602 (2009).

[13] M. Baiesi and C. Maes, New Journal of Physics 15, 013004 (2013).

[14] T. Nemoto and S.-i. Sasa, Physical Review E 84, 061113 (2011).

[15] P. Gaspard, New Journal of Physics 15, 115014 (2013).

[16] C. Y. Gao and D. T. Limmer, Journal of Chemical Physics 151, 014101 (2019).
[17] L. Onsager and S. K. Kim, The Journal of Physical Chemistry 61, 198 (1957).

[18] L. F. Cugliandolo, V. Lecomte, and F. Van Wijland, Journal of Physics A: Mathematical and Theoretical (2019).

[19] U. Basu and C. Maes, in Journal of Physics: Conference Series, Vol. 638 (IOP Publishing, 2015) p. 012001.

[20] D. Frenkel and B. Smit, Understanding molecular simulation: from algorithms to applications, Vol. 1 (Elsevier, 2001).

[21] R. Zwanzig, Nonequilibrium statistical mechanics (Oxford University Press, 2001).

[22] The conductivity to second order in the field is $\frac{\sigma(E)}{\sigma(0)} \approx 1+$ $\frac{\beta^{2} t_{\mathrm{N}}^{2} E^{2}}{8\left\langle J^{2}\right\rangle_{0}}\left(\left\langle J^{4}\right\rangle_{0}-3\left\langle J^{2}\right\rangle_{0}^{2}+3\left\langle J^{2} Q^{2}\right\rangle_{0}-3\left\langle J^{2}\right\rangle_{0}\left\langle Q^{2}\right\rangle_{0}\right)$.

[23] Supplemental Material.

[24] S. Plimpton, Journal of computational physics 117, 1 (1995).

[25] H. S. Harned and B. B. Owen, Journal of The Electrochemical Society 106, 15C (1959).

[26] J. Dzubiella, G.P. Hoffmann, and H. Löwen, Physical Review E 65, 021402 (2002).

[27] N. Kavokine, S. Marbach, A. Siria, and L. Bocquet, Nature nanotechnology 14, 573 (2019).

[28] W. Wilson, The theory of the Wien effect for a binary electrolyte, Ph.D. thesis, $\mathrm{PhD}$ Thesis, Yale University (1936).

[29] P. G. Wolynes, Annual review of physical chemistry 31, 345 (1980).

[30] V. Démery and D. S. Dean, Journal of Statistical Mechanics: Theory and Experiment 2016, 023106 (2016).

[31] J.-P. Péraud, A. J. Nonaka, J. B. Bell, A. Donev, and A. L. Garcia, Proceedings of the National Academy of Sciences 114, 10829 (2017).

[32] J. W. Daily and M. M. Micci, The Journal of Chemical Physics 131, 094501 (2009).

[33] E. Heid and C. Schröder, Physical Chemistry Chemical Physics 20, 5246 (2018).

[34] R. R. Netz, Phys. Rev. Lett. 91, 138101 (2003). 